\title{
The British system for anticoagulant control and Thrombotest
}

\author{
L. POLlER, JEAN M. THOMSON, AND M. R. ALDERSON
}

From the Haematology Department, Withington Hospital, Manchester, and the Department of Social and Preventive Medicine, University of Manchester

SYNOPSIS A study has been made to determine whether a reliable comparison can be made between the British Comparative Thromboplastin and Thrombotest using the nationally adopted standardization procedure. The method recommended appeared sufficiently satisfactory and statistically val id for the comparison between the British Comparative Thromboplastin and Thrombotest.

The degree of diversion in the location of the 'best lines', which should have been in an identical position at each centre, suggests that the standardization procedure at individual hospitals with Thrombotest is not usually a sufficiently reliable basis for clinical anticoagulant dosage. The degree of diversion almost certainly arises from technical error in the standardization procedure, and it is suggested that Thrombotest should be calibrated against the British Comparative Thromboplastin only in specialist coagulation centres thoroughly conversant with both techniques.

The national system for anticoagulant control, introduced in 1969, has been shown to be statistically valid when comparing one type of thromboplastin reagent with another using the Quick prothrombin time technique (Alderson, Poller, and Thomson, 1970). In a recent study of the interpretation of prothrombin results some difficulty was encountered at hospitals using the modified prothrombin technique with Thrombotest (Poller and Thomson, 1969). The present study was designed to determine whether hospitals which used the British Comparative Thromboplastin (BCT) as a reference preparation were able to perform the recommended standardization procedure with Thrombotest. For comparison a Quick test thromboplastin made from rabbit brain was also included in our study. The opportunity was also taken of comparing the results with our recent report on the statistical validity of the nationally adopted standardization procedure using the Quick test (Alderson et al, 1970).

\section{Method of Study}

Two groups of hospitals performed the following studies. In group 1 the recommended standardization procedure was performed with Thrombotest and in group 2 a rabbit brain thromboplastin (Diagen phenolized) was substituted for the British Comparative Thromboplastin and tested in parallel with Thrombotest.

Received for publication 30 July 1970.

\section{GROUP 1}

The same numbered batch (934) of Thrombotest reagent was sent, together with sufficient $3.2 \mathrm{mM}$ calcium chloride solution and a fresh supply of the British Comparative Thromboplastin, to 20 hospitals. Each hospital was asked to test in parallel 20 patients who had been stabilized on oral anticoagulant treatment. They were also asked to test two fresh normal plasma specimens on each day they tested the coumarin specimens. The mean of the normals was used for the determination of prothrombin ratios.

Ten of the 20 hospitals invited to participate were known to use Thrombotest routinely, the other 10 used the Quick test. This was to discover if error resulted from unfamiliarity with the different technique using Thrombotest.

The detailed instructions were as follows:

Hospitals were asked to collect $4.5 \mathrm{ml}$ of venous blood into $0.5 \mathrm{ml}$ of $3.13 \%$ trisodium citrate in a plastic or siliconed container. Specimens were to be tested within one hour of collection where possible and if more than two hours old were not to be used. Blood was to be stored where necessary, at $4^{\circ} \mathrm{C}$. To standardize contact activation each blood sample was to be tested individually with both reagents in turn. Results were recorded as clotting time in seconds. The procedure for Thrombotest was according to the manufacturer's instructions for the venous technique. An instruction sheet was also sent with the details of the Quick test procedure using the British Comparative Thromboplastin. 
GROUP 2

The instructions were as above but the same batch of rabbit brain (Diagen phenolised) was substituted for the British Comparative Thromboplastin and sent to 14 hospitals, seven of which used Thrombotest routinely, the other seven the Quick test. The manufacturer's instructions were enclosed with the rabbit brain together with the recommended method for the British Comparative Thromboplastin.

The prothrombin ratios were calculated by us and we also plotted each set of results on arithmetical graph paper. We then drew the 'best straight line' between all the points by visual assessment in the usual way. Linear regression analysis was carried out and a calculated line inserted on the same graph.

It is important to determine whether the 'best straight line' is a fair representation of the data and the results were analysed from two points of view.

The difference between the 'best straight line' drawn by visual assessment and the calculated line was examined using the present results to see if there was a significant difference and also compared with the results from our recent study (Alderson et al, 1970), when the same method of comparison was used with two different Quick test procedures. The reliability of the 'best line' drawn by visual means was assessed by measuring the distance between the two lines at various points. Seven points with the following values on the scale of the Thrombotest and rabbit brain method were used: ratios of 1.8 , $2 \cdot 0,2 \cdot 2,2 \cdot 4,2 \cdot 6,2 \cdot 8$, and $3 \cdot 0$. In addition the vertical distance between the two lines was measured from the points on the calculated line equivalent to 1.8 and 3.0 with the British Comparative Thromboplastin. The latter two points are the usual extremes of the therapeutic scale with the British Comparative Thromboplastin.

The same batches of thromboplastin reagent were supplied to the hospitals and in theory the location of the lines should therefore not vary from centre to centre in each group. Any deviation almost certainly would represent individual differences in technique and a great variation with one type of reagent or technique would cast doubt on the reliability of the system when this was used. As a method of assessment in group 1, the ratios were recorded for Thrombotest equivalent to 3.0 on the British Comparative Thromboplastin scale. (This is the upper limit of the therapeutic range of the British Comparative Thromboplastin.) In group 2 Diagen was substituted for the British Comparative Thromboplastin. In our previous study (Alderson et al, 1970), using the same batch of rabbit brain, we found the ratio which corresponded to 3.0 on the British Comparative Thromboplastin scale was 2.23 for rabbit brain. The distribution of Thrombotest results equivalent to a Diagen
L. Poller, Jean M. Thomson, and M. R. Aldersono

ratio of 2.23 was obtained using the calculated lines $\stackrel{\bar{O}}{\mathrm{O}}$ This was used as a measure of the scatter of the lines which could be compared with the measure of scatter? for results in group 1.

\section{Results}

1 ANALYSIS OF RETURNS Of the 20 hospitals in the first group, 15 sets of results were regarded as suitable for statisticale analysis. The reasons for exclusion of the five others were as follows:

Four centres tested insufficient numbers of patient and one hospital did not perform the Thrombotes? procedure in the way recommended. Of the $15+$ hospitals, eight of these used Thrombotest routinely the other seven using the Quick test. In the second group statistical analysis was performed on 11 setso of results. Three hospitals had to be excluded. because of incomplete data. Of the 11 analysed, five used Thrombotest as the routine method, the othen six used a Quick test technique.

ANALYSIS OF RESULTS

The first point to be studied was the reliability of the line drawn by visual assessment when the British Comparative Thromboplastin was compared with Thrombotest. Table I gives the mean discrepancyo between the two lines using both of these methods of̂․ assessment. Using the seven values for Thrombotes $\mathbb{R}$ the overall discrepancies for the 12 centres varied between 0.04 and 0.36 , with an average of $0 \cdot 15$. For 3 the two therapeutic limits of the British Comparative Thromboplastin the mean discrepancy varied between 0.03 and 0.37 with an average of 0.17 . The mean discrepancy at the upper and lower limits of the therapeutic range for all centres in each of the threes

\begin{tabular}{|c|c|c|}
\hline Hospital & $\begin{array}{l}\text { Mean Discrepancy } \\
\text { at Seven Values for } \\
\text { Thrombotest }\end{array}$ & $\begin{array}{l}\text { Mean Discrepancy at } \\
\text { Upper and Lower Limits } \\
\text { of Therapeutic Range } \\
\text { for BCT }\end{array}$ \\
\hline 1 & 0.22 & 0.19 \\
\hline 2 & 0.31 & 0.18 \\
\hline 3 & 0.04 & 0.07 \\
\hline 4 & 0.36 & 0.37 \\
\hline 5 & 0.08 & $0 \cdot 35$ \\
\hline 6 & 0.07 & 0.05 \\
\hline 7 & 0.09 & 0.08 \\
\hline 8 & $0 \cdot 21$ & 0.30 \\
\hline 9 & 0.09 & 0.03 \\
\hline 10 & 0.11 & $0 \cdot 20$ \\
\hline 11 & 0.06 & 0.07 \\
\hline 12 & 0.17 & $0 \cdot 22$ \\
\hline 13 & 0.18 & 0.34 \\
\hline 14 & 0.07 & 0.05 \\
\hline 15 & $0 \cdot 18$ & 0.11 \\
\hline Mean & $0 \cdot 15$ & $0 \cdot 17$ \\
\hline
\end{tabular}

Table I Discrepancy between visual line and calculated regression line with Thrombotest compared with British Comparative Thromboplastin (BCT) 
comparisons was: local human brain $0 \cdot 10$, rabbit brain $0 \cdot 15$, Thrombotest $0 \cdot 17$. One-way analysis of variance indicates that the difference between these three means is significant at the $1 \%$ level $(\mathrm{VR}=19 \cdot 1$, $D F=2$ and $35, P<0.01)$. Though the mean discrepancy for Thrombotest is largest for the three comparisons it is not very different from that for rabbit brain; both of these are considerably larger than the mean difference for the local human brain comparison reported in our previous survey (Alderson et al, 1970).

In order to test whether the discrepancies result from a difference in method and not from technical errors, a further comparison was made between a rabbit brain Quick test reagent and Thrombotest in hospitals in group 2. Table II indicates the discrepancy between the plotted line and the calculated regression line for the comparison between Thrombotest and rabbit brain for the 12 centres that participated in this comparison. Table II shows a mean discrepancy for the seven values of Thrombotest which lay between 0.01 and 0.11 with an average of 0.06 . The mean discrepancy for the therapeutic limits for rabbit brain varied between 0.01 and 0.23 with an average of $0 \cdot 1$. Thrombotest and the British Comparative Thromboplastin had shown an average difference of $0 \cdot 17$. A t test suggests that these results are not significantly different $(t=1 \cdot 83, D F=24$, $\mathbf{P}<0 \cdot 1)$ indicating the reliability of the principle of drawing the 'best lines' in this comparison.

\begin{tabular}{lll}
\hline Hospital & $\begin{array}{l}\text { Mean Discrepancy at } \\
\text { Seven Valuesfor } \\
\text { Thrombotest }\end{array}$ & $\begin{array}{l}\text { Mean Discrepancy at } \\
\text { Upper and Lower Limits } \\
\text { of Therapeutic Range } \\
\text { for Rabbit Brain }\end{array}$ \\
\hline 16 & & 0.06 \\
17 & 0.02 & 0.15 \\
18 & 0.10 & 0.14 \\
19 & 0.03 & 0.02 \\
20 & 0.03 & 0.02 \\
21 & 0.03 & 0.07 \\
22 & 0.07 & 0.07 \\
23 & 0.02 & 0.23 \\
24 & 0.11 & 0.20 \\
25 & 0.11 & 0.01 \\
26 & 0.01 & 0.12 \\
Mean & 0.09 & 0.10 \\
\hline
\end{tabular}

Table II Discrepancy between visual line and calculated regression line with Thrombotest compared with rabbit brain

The second and more important aspect from the point of view of anticoagulant dosage is the reproducibility of the results at different centres. Samples of the same batches of reagents were sent to the ceritres operating in both groups. In theory the actual lines should be identical for each of the centres. For any fixed point on the vertical axis the same

\begin{tabular}{lll}
\hline Hospital & \multicolumn{2}{l}{ Estimated Thrombotest Ratio } \\
\cline { 2 - 3 } & Calculated Line & Visual Line \\
\hline 1 & $3 \cdot 76$ & $3 \cdot 85$ \\
2 & $2 \cdot 70$ & $3 \cdot 07$ \\
3 & $3 \cdot 30$ & $3 \cdot 45$ \\
4 & $2 \cdot 89$ & $2 \cdot 45$ \\
5 & $8 \cdot 25$ & $6 \cdot 20$ \\
6 & $4 \cdot 14$ & $4 \cdot 16$ \\
7 & $2 \cdot 88$ & $3 \cdot 20$ \\
8 & $3 \cdot 37$ & $4 \cdot 25$ \\
9 & $3 \cdot 14$ & $3 \cdot 38$ \\
10 & $3 \cdot 26$ & $3 \cdot 70$ \\
11 & $3 \cdot 52$ & $3 \cdot 79$ \\
12 & $3 \cdot 36$ & $3 \cdot 17$ \\
13 & $3 \cdot 54$ & $3 \cdot 12$ \\
14 & $2 \cdot 53$ & $2 \cdot 47$ \\
15 & $3 \cdot 45$ & $3 \cdot 45$ \\
Range & $2 \cdot 53-8 \cdot 25$ & $2 \cdot 47 \cdot 6 \cdot 20$ \\
\hline
\end{tabular}

Table III Thrombotest value at each centre for British Comparative Thromboplastin ratio of 3.0.

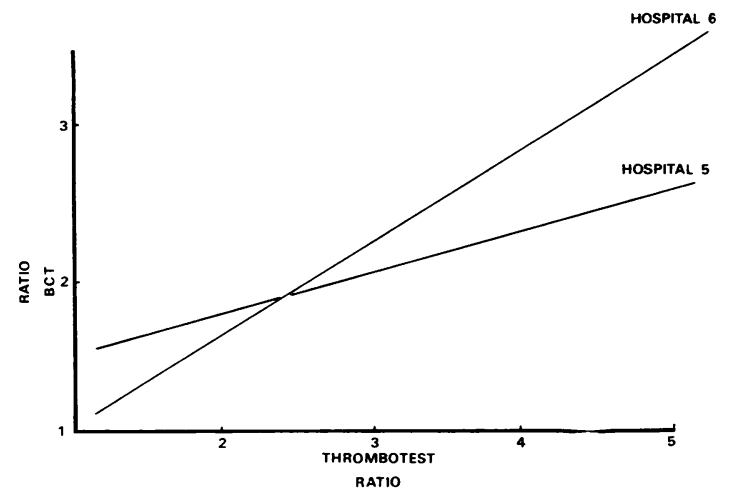

Fig. 1

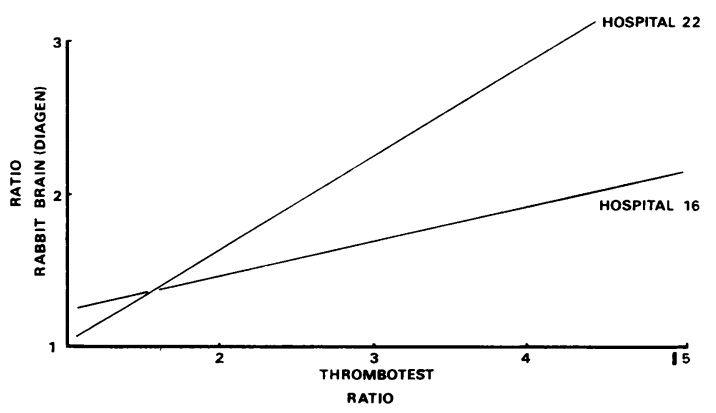

Fig. 2

Figs. 1 and 2 Variation in location of the lines drawn by visual assessment using both the British Comparative Thromboplastin (Fig. I) and

Diagen (Fig. 2) v Thrombotest. 
reading should be obtained on the horizontal axis for each hospital.

In the first group the range of values equivalent to 3.0 on the scale of the British Comparative Thromboplastin is seen to vary between 2.53 and 8.25 on the calculated line $(2.47$ and 6.20 on the line drawn through the points by visual assessment as shown in Table III). Figures 1 and 2 provide examples of this variation in location of the lines drawn by visual assessment using both the British Comparative Thromboplastin $\mathrm{v}$ Thrombotest and Diagen vThrombotest comparisons. On each occasion the line was based on points with a near linear distribution. Table IV shows the values for Thrombotest ratios that appear equivalent at the different centres to a Diagen rabbit brain ratio of $2 \cdot 23$, which is the upper limit of the therapeutic range.

\begin{tabular}{ll}
\hline Hospital & $\begin{array}{l}\text { Estimated Thrombotest } \\
\text { Value (Calculated Line) }\end{array}$ \\
\hline 16 & 5.95 \\
17 & 3.48 \\
18 & $5 \cdot 13$ \\
19 & 3.15 \\
20 & 3.44 \\
21 & 3.03 \\
22 & 2.98 \\
23 & 2.93 \\
24 & 3.40 \\
25 & 4.03 \\
26 & 3.07 \\
Range & $2.93-5.95$ \\
\hline
\end{tabular}

Table IV Thrombotest value at each centre for rabbit brain ratio of 2.3

\section{Discussion}

In our previous paper (Alderson et al, 1970) we showed that the recommended standardization procedure based on the national reference thromboplastin was statistically valid. The present study was designed to determine whether the same procedure would be valid with the Thrombotest technique which is a modification of the prothrombin time test. It appears from this study that when the calculated line was compared with the line drawn by visual assessment through the points obtained by plotting the prothrombin ratios, there was a significant degree of error introduced when the Thrombotest technique was used compared with human brain comparison in the Quick test method. These results, although statistically significant, were probably not of great clinical importance in drawing the 'best line' because there is usually a discernible trend of the points which indicates the correct position of the 'best line' even though the points may not lie as closely to the line with the Thrombotest correlation. In other words the standardization procedure recommended with the British Comparative Thrombo- plastin is sufficiently satisfactory and statistically $\stackrel{0}{\overrightarrow{0}}$ valid for the comparison between the British com-. parative thromboplastin and Thrombotest technique $\overrightarrow{\overline{\mathrm{F}}}$ as well as for the British Comparative Thromboplastinand Quick test reagents.

More relevant clinically was the large variation in $\frac{\overline{\bar{c}}}{\overline{5}}$ points equivalent to a prothrombin ratio of 3 with $\vec{\nabla}$ the British Comparative Thromboplastin, ie, the upper limit of the therapeutic range (see Table III). ळ This is also shown by the location of the lines when $\vec{\circ}$ Thrombotest was compared with either of the Quick $\overrightarrow{-}$ test reagents. As the hospitals received the same $\vec{\omega}$ numbered batch of British Comparative Thrombo-긍 plastin, Thrombotest, and Diagen in the present trial, in theory their results should have been identical $\perp$ from centre to centre, and the degree of scatter of the? ratios equivalent to 3 with the British Comparative $\vec{A}$ Thromboplastin is a measure of the reproducibility ${ }_{0}^{\omega}$ of technique at the individual hospitals. The wide ${ }^{-}$ distribution of readings corresponding to $2 \cdot 23 \vec{z}$ Diagen, which we have shown to be equivalent too 3.0 on the British Comparative Thromboplastin scale $\mathrm{S}$ (Table IV), indicates that the difficulties encountered $\vec{A}$ with Thrombotest cannot be explained by the $\stackrel{\varphi}{v}$ differences between human brain and animal brain.thromboplastin as both Diagen Quick test reagent and Thrombotest are made from animal brain extract. The only possible explanation is that the failure to obtain reproducible results with the stand-气 ardization procedure, when Thrombotest is com- $\stackrel{\mathbb{Q}}{\varrho}$ pared with the British Comparative Thromboplastin, $\overrightarrow{\vec{B}}$ is due to differences in laboratory technique. Few 3 hospitals are sufficiently conversant with the technical pitfalls of both methods for an accurateo comparison. The degree of diversion of the lines at the different hospitals was so great that one must? question the feasibility of standardizing results using the nationally adopted standardization procedure. at laboratories using Thrombotest. The present studyo suggests that it would be unwise to base patients? anticoagulant dosage on this 'standardization pro-음 cedure' with Thrombotest except perhaps in a small number of expert coagulation laboratories. The reliance on a few 'expert' centres is not as good orr reliable as performing the routine standardization procedure oneself, because local variations in technique in individual laboratories will not be con- $\omega$ trolled. There can be no certainty that the resulting British Corrected Ratio on iwhich anticoagulanto dosage depends is accurate.

\section{References}

Alderson, M. R., Poller, L., and Thomson, J. M. (1970). Validity of the British System for anticoagulant control using the? national reagent. $J$, clin. Path, 23, 281-285.

Poller, L., and Thomson, J. M. (1969). The interpretation of prothrom bin results. A national survey. Brit. J. Haemat., 16, 31-37. 